\title{
PEDIATRIC NURSE PRACTITIONER HOME VISITATION IS ASSOCIATED WITH A DECREASE IN HOME CARE AND HEALTHCARE UTILIZATION ERRORS IN HIGH RISK INFANTS
}

\author{
By Rupalee Patel
}

A doctoral project in partial fulfillment of the requirements for the degree of Doctorate of Nursing Practice in the California State University, Northern Consortium, Doctor of Nursing Practice Program, California State University, Fresno

May 2015 


\section{APPROVED}

For the Department of Nursing:

We, the undersigned, certify that the thesis of the following student meets the required standards of scholarship, format, and style of the university and the student's graduate degree program for the awarding of the doctoral degree.

Rupalee Patel

Doctoral Project Author

Daryl Canham

Nursing

Chairperson's name (Chair)

Department name

Balaji Govindaswami

Pediatrics

Committee member's name

Department name

Korinne VanKeuren

Pediatrics

Committee member's name

Department name

or professional affiliation

For the University Graduate Committee:

Dean, Division of Graduate Studies 


\title{
AUTHORIZATION FOR REPRODUCTION OF DOCTOR OF NURSING PRACTICE PROJECT
}

\author{
I, Rupalee Patel grant permission for the reproduction of this project (Pediatric Nurse \\ Practitioner Home Visitation is Associated with a Decrease in Home Care and Healthcare \\ Utilization Errors in High Risk Infants) in part or in its entirety without further authorization \\ from me, on the condition that the person or agency requesting reproduction absorbs the cost and \\ provides proper acknowledgment of authorship.
}

Signature of DNP project author:

Date: 


\section{Dedication}

This doctoral project is dedicated to my mother and father, Bina and Vishnu Patel, who have sacrificed themselves to help me rise and who have supported me every step of the way.

\section{Acknowledgements}

First I would like to thank my husband Shane, sweet baby Anjali, Mom, Dad and little sister Gopi for their love and support throughout my doctoral program. You gave me strength, love and patience when I needed it the most. I could never have done this without all of you.

I would like to thank my clinical mentor Dr. Balaji Govindaswami for his support, guidance and the amazing opportunities that he has provided me. I would like to thank my doctoral advisor, Dr. Ruth Rosenblum for all of her help and support throughout the doctoral program. I would like to thank my project chair Dr. Daryl Canham for believing in me. I would like to thank my friend and mentor Dr. VanKeuren for inspiring me. Dr. Priya Jegatheesan, Dr. Sunshine Weiss and Dr. Dongli Song thank you for taking me under your wings.

Finally, I would like to thank the infants and their caregivers for allowing SCVHHS BRIDGE PNPs into their lives and homes, SCVHHS Administration, SCVHHS NICU multidisciplinary team, SCVHHS Ambulatory Care Pediatric providers, and Santa Clara County Public Health staff, First 5 Santa Clara County and the Valley Medical Center Foundation for their ongoing support. Without them, there would be no project. 
Pediatric Nurse Practitioner Home Visitation is Associated with a Decrease in Home Care and Healthcare Utilization Errors in High Risk Infants Rupalee Patel, DNPc, MS, BSN, C-PNP, C-PHN, IBCLC California State University, Northern California Consortium Doctor of Nursing Practice School of Nursing May $1^{\text {st }}, 2015$ 


\begin{abstract}
The Santa Clara Valley Health and Hospital System’s (SCVHHS) Babies Reaching Improved Development and Growth in their Environment (BRIDGE) program was developed in 2011 to optimize high risk infants' care transition from Neonatal Intensive Care Unit (NICU) to home. In addition to hospital discharge teaching and public health nursing efforts, NICU infants need further in home support given their medical vulnerability after discharge. The objectives of the SCVHHS BRIDGE program are to provide caregiver interventions to minimize home care errors after NICU discharge and to optimize health care access and utilization across the transition of care. SCVHHS NICU infants at risk for developmental delay, who met Department of Health and Human Services (DHHS) California Children's Services (CCS) High Risk Infant Follow-up (HRIF) criteria, qualified for SCVHHS BRIDGE visits. From April of 2011 to January of 2015 Pediatric Nurse Practitioners (PNPs) met caregivers in the NICU, visited homes a minimum of two times after discharge, identified errors, educated caregivers, coordinated care, and facilitated in the resolution of the errors. Errors were defined as a deviation from prescribed plan of care upon discharge from the NICU or after an ambulatory care visit. Data were collected prospectively and Institutional Review Board (IRB) approved. Chi Square with Yates correction was used to assess for significance. Wilcoxon Rank Sum test was used to assess association between gestational age, length of stay, and birth weight in infants with and without errors. Wilcoxon Signed Rank test was used to assess for significance of error reduction across visits. PNPs monitored, educated and attenuated home care errors between home visits and caregivers over time. Collaborating with caregivers and health care providers in the inpatient, home, and outpatient environments, PNPs have the potential to decrease errors, improve health, reduce health care costs, and optimize growth and development for high risk infants.
\end{abstract}


Table of Contents

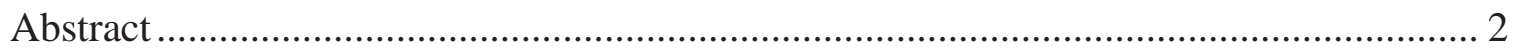

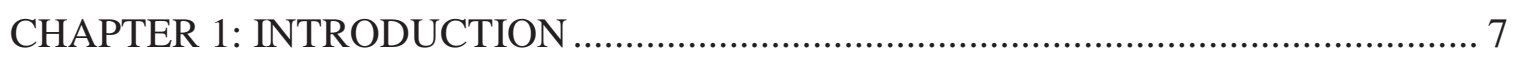

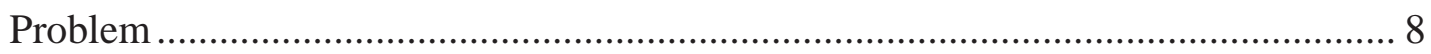

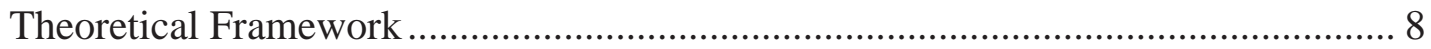

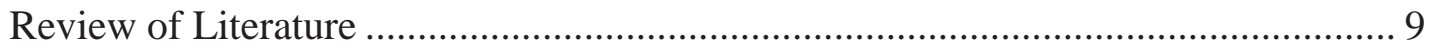

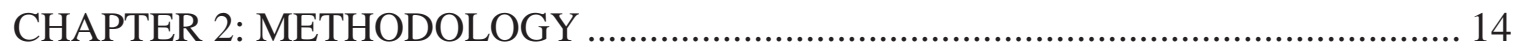

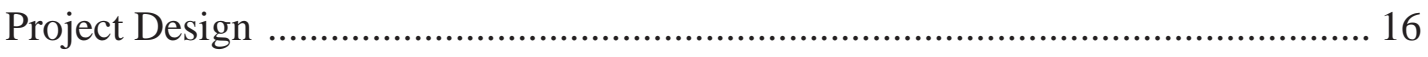

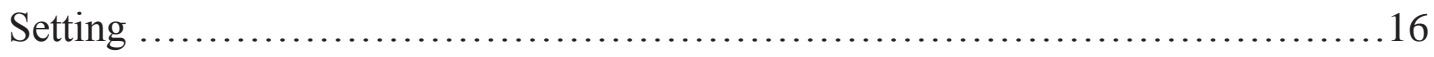

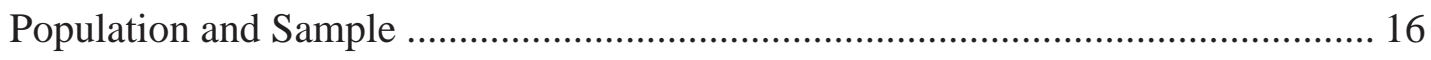

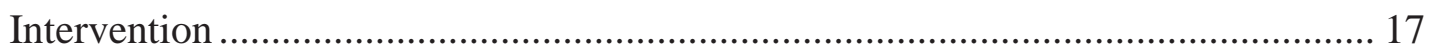

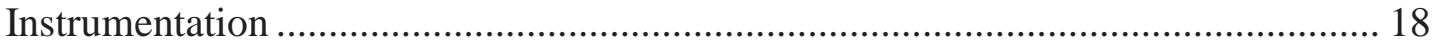

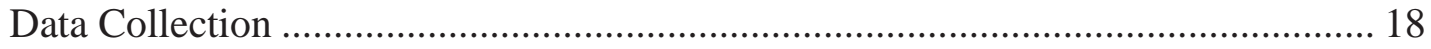

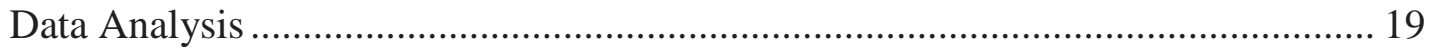

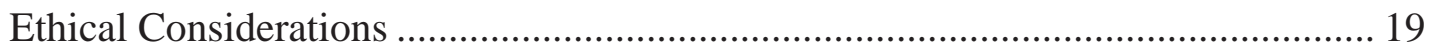

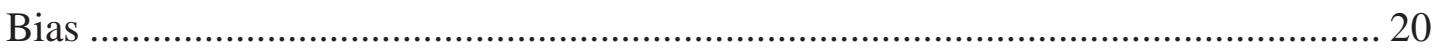

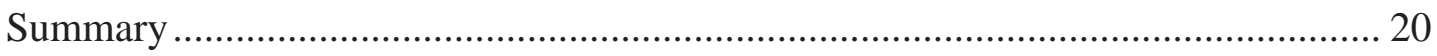

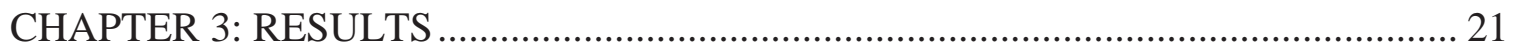

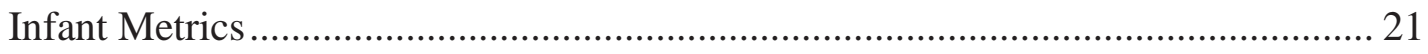

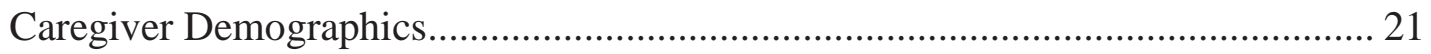

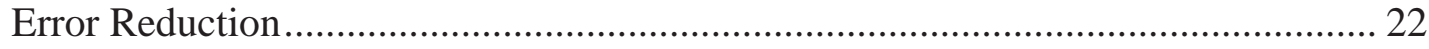

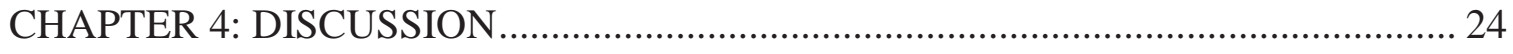

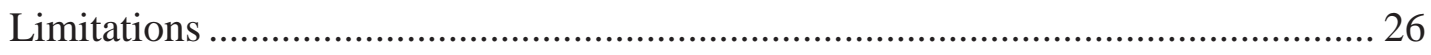

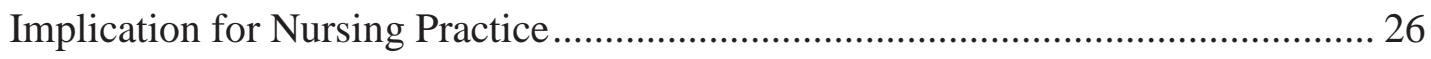

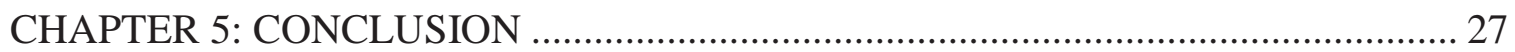

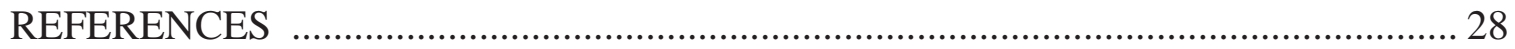


APPENDIX A: CCS HRIF Program Medical Eligibility Criteria ........................................ 33

APPENDIX B: SCVHHS BRIDGE Data Collection Sheet .............................................. 34 


\section{LIST OF TABLES}

Table 1. Categorization of High Risk Infants ............................... 17

Table 2. Infant Metrics ..................................................21

Table 3. Primary Caregiver Demographics .................................22

Table 4. Number of Errors ...........................................23 


\section{LIST OF FIGURES}

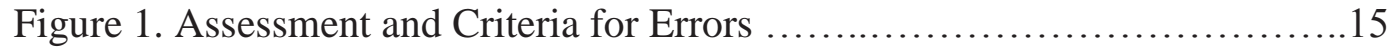

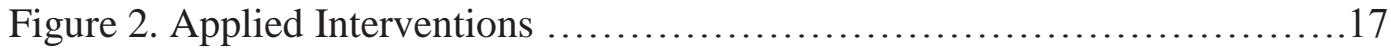

Figure 3. Graphical Representation of Error Reduction Over Visits .............23 
PEDIATRIC NURSE PRACTITIONER

Pediatric Nurse Practitioner Home Visitation is Associated with a Decrease in Home Care and Healthcare Utilization Errors in High Risk Infants

\section{Introduction}

The Centers for Disease Control and Prevention National Center for Health Statistics has identified that approximately 500,000 premature infants are born in the United States every year (Martin \& Osterman, 2013). The infants who are born less than 32 weeks gestation and who weigh less than 1500 grams at birth are especially at risk for developmental delay based on CCS high risk infant medical eligibility criteria (High Risk Infant Follow-up Quality of Care Initiative, 2012). The World Health Organization and the United Nations International Children's Emergency Fund recommend that home visits be implemented by trained personnel as an intervention to decrease infant morbidity and mortality (World Health Organization, 2009). SCVHHS BRIDGE program was developed and implemented in April of 2011 to optimize the HRIs' care transition from the NICU to home in hopes of facilitating health care maintenance and anticipatory guidance.

The Institute of Medicine in collaboration with the Robert Wood Johnson Foundation recommends wider use of advanced practice nurses in home care. Home visits by nurse practitioners who may provide primary care services, care coordination services, non-emergent medical services and triage services may help to decrease unnecessary emergency room visits and may increase access to primary care services that may help to potentially decrease morbidity and mortality in the high risk infant post hospital discharge. Home visits by nurse practitioners who bridge inpatient and outpatient infant needs has the potential to improve access to primary health care, reduce health care costs, improve parent-infant interactions, and may result in the optimal growth and development for premature infants. 


\section{Problem}

High risk infants (HRI's) are discharged from the NICU with complex and dynamic medical needs that require continued comprehensive care. Families struggle to become competent in areas including: medication administration; complex feeding preparation; and appropriate use of medical equipment. For HRI's a caregiver's lack of understanding with regard to feedings, medications, or medical equipment in the NICU post discharge period can have a negative impact on the infant's health status (Hulsey, Hudson and Pittard, 1994). Inappropriate dosing and or mixing of medications or specialty formulas as well as inability to use medical equipment correctly can result in an increase in morbidity and mortality in the HRI (Hulsey, Hudson and Pittard, 1994). Therefore, this Doctor of Nursing Practice (DNP) project focus will be to study the early effectiveness of the SCVHHS BRIDGE program by examining whether PNP home visitation for HRI's is associated with a reduction in homecare and healthcare utilization errors.

\section{Theoretical framework}

Orem's theory of self-care deficit is a framework that captures the essence of the SCVHHS BRIDGE program. The self-care deficit theory (SCDT) is a middle range nursing theory that specifies when nursing intervention is needed with regard to patient's self-care. According to Orem, nursing is required when a patient is incapable or limited in the provision of continuous effective self-care (Hartweg, 1991). Orem's theory of self-care deficit identified 5 methods that compose the basis of the theory. These 5 methods are: acting for and doing for others, guiding others, supporting another, teaching another and providing an environment promoting personal development in relation to meet future demands (Hartweg, 1991). All components are integral with regard to the SCVHHS BRIDGE program's PNP's duties. 
Orem's SCDT was chosen to support the DNP project focus over other health behavior theories because Orem's SCDT focused on promoting continuous effective care through nursing guidance whereas other health belief theories such as the theory of planned behavior and the trans-theoretical model and stages of change focus on healthcare promotion for treatment adherence (Glanz, Burke, Rimer, 2011). Orem's SCDT provides a model for articulation of appropriate, timely and sequenced actions that are required to achieve health care maintenance (Taylor \& Cox, 2005). The overall goal of Orem's SCDT is to promote life, health, development and well-being (Banfield, 2011). The ultimate goal of the SCVHHS BRIDGE program is to help the caregivers to promote life, health, development and well-being of the high risk infant. The SCDT focuses on nurses bringing about new or changed conditions in the patient and the patient's environment in order to meets the patient's therapeutic self-care demand and to regulate the development and exercise of the patient's self-care agency (Berbiglia, 2011). The SCVHHS BRIDGE program is able to provide highly individualized interventions through education to the caregivers of the high risk infant to help the infant meet his or her therapeutic self-care demands through the utilization of PNP's.

SCVHHS BRIDGE program PNP's primary purpose is to serve the family and ease the transition from the NICU to the home by providing health care maintenance and anticipatory guidance measures as well as health promotion. Orem's SCDT is a framework that captures the spirit of the SCVHHS BRIDGE program.

\section{Review of literature}

Upon reviewing the literature, no studies were found which addressed medication, feeding, equipment or appointment errors in high risk infants as defined by DHHS CCS HRIF 
eligibility criteria during the transition from the NICU to home. Thus, the following review is based on articles that share a common thread with the doctoral projects purpose in the domains of home care and healthcare utilization as well as advanced practice nurses and high risk infants.

Medication administration errors not only occur when the patient is in the NICU but also occur when the patient is discharged home with medications. John (2005) explored the various causes for medication errors in the pediatric population within their home settings and provided suggestions on educating the patient's caregivers on the prevention of medication errors. Although recommendations were provided to caregivers to prevent medication errors the need for reinforcement of medication administration once the patient was discharged home was not addressed (John, 2005). Based on John's (2005) findings, a home follow-up program such as SCVHHS BRIDGE program can act as an avenue to promote behavior change in the caregiver of the HRI by providing in home reinforcement with regard to medication administration.

Feeding preparation errors not only occur when the patient is in the NICU but also occur when the patient is discharged home with specialized feeding preparations. Evans et al. (2012) found that patients discharged home with complex feeding preparations administered via enteral feeding tube had a decrease in the correct feeding preparation. Thus Evans et al. (2012) recommended that caregiver skills related to feeding preparation of enteral fed HRI's should be re-assessed once the patient is at home. SCVHHS BRIDGE program PNP's have the caregiver of the HRI demonstrate how the feeding is prepared to ensure proper preparation.

Medical equipment use errors not only occur when the patient is in the NICU but also occur when the patient is discharged home with medical equipment. Evans et al. (2012) also found that enteral feeding tubes were not properly being used by the caregiver once the patient was discharged home. Due to their findings, Evans et al. (2012) recommended that in home 
support is provided to caregivers on an annual basis to ensure proper use of enteral feeding tubes. SCVHHS BRIDGE program PNP's assess all medical equipment that the patient is discharged home with and ensure that the caregiver knows how to properly use medical equipment Sparks (2009) examined the associations among socio-demographic characteristics, health care access, maternal health status, and health behaviors influence on birth weight disparities among several racial/ethnic groups. Findings suggested that differences associated with health care access and health behaviors led to a variation in infant health outcomes among a diverse set of racial/ethnic groups. Wang, Guttmann, To, and Dick (2009) examined the effect of socioeconomic status (SES) on health outcomes in 512, 768 infants with complex chronic conditions insured through a universal health plan. The researchers found that despite having universal health coverage, SES related inequality affected hospitalization and morbidity rates among medically vulnerable infants.

A major barrier to the access and utilization of healthcare for vulnerable populations is limited English proficiency (LEP). Yu, Huang, Schwalberg and Nyman (2006) examined the relationship between parental English proficiency and their child's access to health care. Findings suggested children with parents with LEP had greater difficulty with appropriately utilizing and accessing healthcare. They also concluded that language barriers are one of the main causes of lack of knowledge with regard to healthcare promotion and community resources. Due to this lack of knowledge, parents of the HRI who have LEP need a system in place to ease the transition from the NICU to their home. Miquel-Verges, Donohue and Boss (2006) examined the needs of Latino families with LEP whose infants were discharged from the NICU. They interviewed 36 mothers with LEP who had very low birth weight infants in the NICU. Findings suggested that more than half of the mothers were unaware of early developmental intervention 
programs for which their infants were eligible for. In addition, they found that one month after NICU discharge many mothers were unaware of necessary follow-up services or had difficulty obtaining specialty services.

Barriers to accessing healthcare in the HRI can result in increased long term morbidities such as developmental delays (Guralnick, 2011). Barriers to care were identified in order to promote optimal HRI outcomes by Catlett, Thompson, Johndrow and Boshkoff (1993). The researchers examined 130 families and identified several factors associated with loss to followup including: race, level of education, and lack of transportation. In addition to caregiver low SES, Roberts et al. (2007) similarly found that difficulty in accessing early intervention services for the HRI is due to caregiver race (African American or Hispanic) and low education levels. Focus group results concluded that the major barriers with regard to accessing healthcare for underserved children were caregiver low SES, race (African American or Hispanic), low educational attainment and LEP (Ames, 2007). The findings from the studies discussed above share similar barriers with regard to access of healthcare for underserved children, these are caregiver race (African American or Hispanic), low SES, low level of education and lack of transportation.

Once barriers to accessing healthcare are identified, realistic solutions must be proposed and implemented to address them. The barrier of transportation was addressed by Catlett, Thompson, Johndrow and Boshkoff (1993) who found that providing transportation for the HRI to clinic successfully resulted in a decrease in loss of follow-up. Although Roberts et al. (2007) did not implement a specific solution for the barrier of low caregiver education level, they proposed that providing caregivers with educational tools that help identify delays in development may initiate earlier access to early intervention services. Ames (2007) proposed a 
solution of providing a home visiting family advocate to provide education and instituting mobile clinics as well as utilizing public buildings in local neighborhoods to address the barriers of caregiver low SES, low education level and lack of transportation. The challenge to overcoming the major barriers of accessing healthcare for the HRI is to maintain evidence based implemented solutions and implement evidence based proposed solutions similar to the ones provided above.

Nurse practitioner (NP) home visits lead to improved health outcomes and utilization of resources (Neal-Boylan, Mager, \& Kazer, 2012). NP home visits reduce emergency department visits, hospital readmissions and healthcare costs while delivering expert comprehensive care, continuity of care, chronic illness management, and improving transitional care (Brooten et al., 1995; Naylor, Bowles, \& Brooten, 2000; Naylor et al., 1999; Running \& Walker, 1999). The Institute of Medicine recommends a wider use of nurse practitioners in a variety of health care delivery systems, including in the home (Institute of Medicine, 2011; Institute of Medicine, 2003).

PNPs have provided high quality health care to vulnerable populations in the United States since 1964 (Duderstadt, 2008; Murphy, 1990). Due to their scope of practice and knowledge base, PNPS are able to fill a gap in the care of high risk infants across transition that current follow-up providers, primarily pediatricians and public health nurses (PHNs), may be unable to fulfill. While PHNs provide support through home assessments, the increased scope of practice of PNPs allows them to provide additional support, including identifying and correcting in home errors. Providers in a primary care office may have limitations on their time due to their high volume of patients, PNP home visits provide valuable support for high risk infants because the PNP scope of practice includes monitoring and advising caregivers on the overall health of 
their infants, referring families to appropriate healthcare and community services, monitoring infant growth and development, providing anticipatory guidance and health care maintenance, evaluating caregiver needs, prescribing medications, and facilitating ordering of needed medical equipment (Looman et al., 2013;Cady et al., 2014). PNPs can offer targeted education with regard to identified errors in care regimen thus improving transitions of care.

HRI's are at increased risk for morbidity and mortality after hospital discharge compared to healthy term infants (Guralnick, 2011). To decrease this morbidity and mortality high risk infants discharged from NICUs often require individually tailored feeding regimens, in home medical equipment, appropriate medications and a multitude of follow-up appointments (American Academy of Pediatrics, 2008). Caregivers of high risk infants are responsible for carrying out these complex and dynamic care regimens upon NICU discharge, in the home and thus have an increased need for the support provided by home visitation services (Cohen, 1991).

PNP follow-up visits provide an ideal environment for evaluating the prescribed therapeutic regimen at discharge and developing interventions that reduce errors in feeding, medication, equipment and appointments.

\section{Methodology}

PNPs conducted home visits for high risk infants discharged from SCVHHS's regional level IV NICU. The first visit occurred within two weeks of NICU discharge. Subsequent follow up visits were scheduled as needed based on PNP evaluation of medical need until no longer required. At each visit, the PNP reviewed the patient's medical record, conducted a comprehensive history and physical exam, and performed an interactive review consisting of record review, caregiver interview, and observation of return demonstration of feeding preparation and administration, medication administration, and equipment use; assessment of 
needed and scheduled infant follow up appointments was done via medical record review and caregiver interview. During each home visit, the PNP performed a structured assessment and documentation of errors (see figure 1 below). A home care error was defined as an error with regard to feeding, medication or equipment that occurred in the home which resulted from a deviation from prescribed plan of care upon discharge from the NICU or after an ambulatory care visit. A healthcare utilization error was defined as an error with regard to appointments that occurred in the home which resulted from a deviation or absence of a prescribed follow-up appointment plan of care upon discharge from the NICU or after an ambulatory care visit.

Figure 1: Assessment and Criteria for Errors

\begin{tabular}{|clllc|}
\hline \multirow{2}{*}{ Assessment } & Feeding & Medication & Equipment & Appointment \\
& Record review; & Record review; & Record review; & Record review; \\
& Caregiver & Caregiver & Caregiver & Caregiver \\
& interview Return & interview return & interview Return & interview \\
& demonstration & demonstration & demo & \\
Error Criteria & Preparation or & Preparation or & Bulb Suction, & Scheduled but \\
& administration & administration & Thermometer, & missed \\
& errors & errors & other (eg G-Tube, & - Parent \\
& & & Home oxygen, & unaware \\
& & & NGT, Wound & - Parent aware \\
& & & supplies, Anal & Not Scheduled \\
& & dilators, & - By parent \\
& & Measuring spoons) & - By provider \\
\hline
\end{tabular}

PNPs documented home care and healthcare utilization errors in four domains: feeding, medication, equipment and appointment. Examples of feeding errors included incorrect fortification of breastmilk or formula, or administering the wrong type of formula. Medication errors included incorrect and medication errors included incorrect dose, failing to administer or obtain a medication. Equipment use errors consisted of lacking equipment or incorrect use of bulb syringe, thermometer, or other home health care equipment. If the wrong measuring 
instrument was used to prepare a feeding, an error was documented for both preparation of feeding and for equipment. Appointment errors were defined as missing a scheduled appointment or failure to schedule an appointment. Any error that was identified and subsequently recurred was counted as a second error. However, the PNPs did facilitate the PHN or pediatrician in resolution of the error. Only errors that were identified by PNPs in the home and intervened by PNPs in the home were reflected in the data.

\section{Project design}

This is a prospective observational cohort study of DHHS CCS HRIF eligible infants admitted to SCVHHS regional level IV NICU between April 2011 and January 2015.

\section{Setting}

This study was conducted in the homes of high risk infants after discharge from SCVHHS's regional level IV NICU. SCVHHS is a public safety net county hospital serving Santa Clara County's culturally and socio-economically diverse population living in urban, suburban, and rural areas.

\section{Population and sample}

The study included 153 infants who met DHHS CCS, HRIF eligibility criteria (see Appendix A) and received at least two SCVHHS BRIDGE home follow-up program PNP visits after SCVHHS NICU discharge. HRIs are presented based on the DHHS CCS HRIF medical eligibility criteria. Categorization of high risk infants is presented in the table below (see table 1). Infants who died within two weeks of discharge, moved out of the county, were lost to follow up, had only one visit, or whose parents declined visits were excluded from the study. 
Table 1: Categorization of High Risk Infants

\begin{tabular}{|c|c|}
\hline & n (\%) \\
\hline Total HRIF Infants & 153 \\
\hline$<32$ wks OR $<=1500 \mathrm{~g}$ & $109(71 \%)$ \\
\hline$\hookrightarrow<=1500 \mathrm{~g}$ & $52 \%$ \\
\hline$\longrightarrow<32 \mathrm{wks}$ & $63 \%$ \\
\hline$\longrightarrow>=32$ wks AND $<=1500 \mathrm{~g}$ & $8 \%$ \\
\hline$\longrightarrow<32$ wks $\mathrm{AND}>1500 \mathrm{~g}$ & $19 \%$ \\
\hline$>=32$ ws $\mathrm{AND}>1500 \mathrm{~g}$ & $44(29 \%)$ \\
\hline$\hookrightarrow \mathrm{pH}<7$ or low Apgars at birth & $14 \%$ \\
\hline$\hookrightarrow$ Other dx possibly resulting in CNS abnormaility & $10 \%$ \\
\hline$\longrightarrow$ Inhaled Nitric Oxide $>4 \mathrm{hrs}$ & $2 \%$ \\
\hline$\longrightarrow$ Chronic Lung Disease & $1 \%$ \\
\hline$\longrightarrow$ Seizures & $1 \%$ \\
\hline $\begin{array}{l}\longrightarrow \text { Prolonged hypoxia, acidemia, hypoglycemia } \\
\text { requiring pressor support }\end{array}$ & $1 \%$ \\
\hline
\end{tabular}

\section{Interventions}

When PNPs observed an error in the home, they responded by giving caregivers immediate, targeted education, writing prescriptions, making referrals, scheduling visits, and arranging for equipment delivery as needed (see figure 2). PNPs broad scope of practice allows them to provide anticipatory guidance, health care maintenance, evaluation, prescriptions, referrals and comprehensive care coordination to patients and their caregivers.

Figure 2: Applied Interventions

\begin{tabular}{|cllll|}
\multicolumn{1}{c}{ Feeding } & Medication & Equipment & Appointment \\
\hline \multicolumn{1}{|c|}{ PNP } & Demonstration of & Demonstration of & Demonstration, & Scheduled \\
Interventions & correct & correct & coordinate & appointments, \\
& preparation, & preparation, & obtaining & made referrals, \\
& administration. & administration, & equipment with & communicated \\
& Customized & prescribed & suppliers and & appointments and \\
& feeding plan and & medication & caregivers & gave handouts to \\
& gave handouts to & & & caregivers \\
& caregivers. & & & \\
& Prescribed & & & \\
& formula. & & & \\
\hline
\end{tabular}




\section{Instrumentation}

The data collection sheet was developed by the study investigator upon launching the SCVHHS BRIDGE program and discovering common errors that were occurring in the home in the homes of high risk infants and their caregivers early on (see Appendix B). An existing data collection form could not be used since there is not a pre-existing form that has been validated. The data collection form that was used did not affect reliability or validity of the information retrieved due to the collection of data being a standard of practice for the SCVHHS BRIDGE program home health encounter. The recording of errors was included in the SCVHHS BRIDGE program PNPs orientation prior to conducting home visits. The data collection sheet was scored based on the number of errors that were recorded in each error domain.

\section{Data collection}

All data were collected by SCVHHS BRIDGE program PNP's. Data with regard to visit number and identification of appointment, medication, feeding or equipment errors were logged during the home visit on a data collection sheet with no patient identifying information. The data was then transferred to a excel spreadsheet on the password protected SCVHHS system (J Drive). No protected health information (PHI) was transferred to the spreadsheet. The data collection sheets were shredded on a daily basis after entry into the spreadsheet by the investigators. Data was entered in the infant's electronic medical record due to the standard of care for home visit charting and was entered into the password protected data collection excel spreadsheet on the same day post home visit. 


\section{Data analysis}

For categorical variables with regard to infant metrics and maternal demographics for infants with and without errors, Chi Square with Yates correction was used to assess for significant differences. Wilcoxon Rank Sum test was used to look at association between gestational age, length of stay, and birth weight for infants with and without errors. Wilcoxon Signed Rank test was used to assess for significance of error reduction for total number of errors and the individual types of errors in infants across visits. Statistical analysis was done using $\mathrm{R}$ version 3.1.2 (cran.r-project.org).

\section{Ethical considerations}

This study was IRB approved by both SCVHHS and California State University, Fresno human subjects review committees. There were no known potential problems and risks with regard to implementation of this study in infants and their caregivers who have been discharged from the NICU. The potential benefits for the infants and caregivers that are enrolled in the study include but are not limited to improved health and optimized growth and development. The potential benefits to the population of high risk infants may be the development of interventions that are based on the specific errors that are identified in the home thus possibly reducing morbidities. The potential benefits to society would be potential for reduced health care costs by improving access and utilization of healthcare services to the high risk infant. Protected health information was not collected in this study. There was no compensation for the patients because the SCVHHS BRIDGE visit is a routine part of care.

A waiver for obtaining informed consent and authorization under Health Insurance Portability and Accountability Act (HIPAA) was waived because this study was a quality improvement project which involved minimal to no risk to the subjects. The waiver or alteration 
did not adversely affect the rights and welfare of the subjects. Also, any caregivers who requested additional pertinent information about the study or about the SCVHHS BRIDGE Program's effectiveness was provided the information as it was available. A waiver of authorization under HIPAA was approved because no PHI was used in the study. While PHI was used in the coordinating of SCVHHS BRIDGE visits for clinical purposes, no PHI was collected in this study.

\section{Bias}

Due to the fact that data which was obtained was a standard routine part of a SCVHHS BRIDGE home visit, there was very little room for investigator bias. The PNPs utilized a data collection sheet and had a very clear understanding of the definition of home care and healthcare utilization errors. Data was validated by a disinterested third party physician analyst by reviewing patient's electronic medical records of the SCVHHS BRIDGE visit encounter to ensure that errors were not over or under represented in the data reporting. The PNPs performance with regard to identification and reduction of home care and healthcare utilization errors were not tied to an increase in pay or any other rewards. Certified translation services were used when needed to properly assess and intervene errors in order to avoid investigator bias.

\section{Summary}

547 home visits were conducted for 153 high risk infants between 2011 and 2015 There was a median of 3 visits (range of 2-8 visits) per patient. Wilcoxon Signed Rank test was used to assess for significance of error reduction. In the following sections of the paper, study results, discussions, implication for nursing practice and concluding statements will be presented. 


\section{Results}

\section{Infant metrics}

Of the 153 high risk infants, 70 were male. The median gestational age was 31 weeks with an interquartile range (IQR) of 28-34 and the median length of NICU stay was 35 days (IQR 20-72). There was no association between infant error types and gestational age, length of stay, birth weight, or gender (see table 2).

Table 2: Infant Metrics

\begin{tabular}{|ccccc|} 
& $\begin{array}{c}\text { Study Group } \\
(\mathbf{n = 1 5 3 )}\end{array}$ & $\begin{array}{c}\text { Group with } \\
\text { Error } \\
(\mathbf{n = 1 0 5})\end{array}$ & $\begin{array}{c}\text { Group without } \\
\text { Error } \\
(\mathbf{n = 4 8 )}\end{array}$ & P Value \\
\hline Birth Weight, & 1460 & 1460 & 1420 & 0.48 \\
Median (Range) & $(470-4060)$ & $(470-3960)$ & $(500-4060)$ & \\
Gestational Age, & 31 & 30.86 & 31.07 & 0.39 \\
Median (Range) & $(23.71-41.14)$ & $(23.71-41.14)$ & $(24.57-40.43)$ & \\
Length of Stay, & 36 & 35 & 36 & 0.24 \\
Median (Range) & $(3-114)$ & $(4-167)$ & $(3-114)$ & 0.22 \\
Male Gender, \% & $46 \%$ & $42 \%$ & $54 \%$ & \\
\hline
\end{tabular}

\section{Caregiver demographics}

Socio-demographic data was obtained for the 146 primary caregivers taking into account caregivers who had multiple infants. There was no association between home care or healthcare utilization errors and primary caregiver level of education, marital status, primary language, parity, age, race and ethnicity (see table 3). 
Table 3: Primary Caregiver Demographics

\begin{tabular}{|c|c|c|c|c|c|}
\hline & & $\begin{array}{c}\text { Primary } \\
\text { Care } \\
\text { Giver } \\
\end{array}$ & $\begin{array}{l}\text { Group with } \\
\text { Errors (\%) }\end{array}$ & $\begin{array}{c}\text { Group } \\
\text { without } \\
\text { Errors (\%) }\end{array}$ & $\begin{array}{c}\mathbf{P} \\
\text { Value }\end{array}$ \\
\hline \multirow{2}{*}{$\begin{array}{l}\text { Ethnicity } \\
(n=146)\end{array}$} & Hispanic & $64 \%$ & $64 \%$ & $65 \%$ & \multirow{2}{*}{0.93} \\
\hline & Non Hispanic & $36 \%$ & $36 \%$ & $35 \%$ & \\
\hline \multirow{4}{*}{ Race $(n=146)$} & White & $77 \%$ & $79 \%$ & $76 \%$ & \multirow{2}{*}{0.85} \\
\hline & Non White & $23 \%$ & $21 \%$ & $24 \%$ & \\
\hline & Black & $3 \%$ & $2 \%$ & $4 \%$ & \\
\hline & Asian & $16 \%$ & $17 \%$ & $15 \%$ & \\
\hline \multirow{2}{*}{ Age $(n=146)$} & $<19$ & $3 \%$ & $4 \%$ & $10 \%$ & \multirow{2}{*}{0.21} \\
\hline & $>=19$ & $97 \%$ & $96 \%$ & $90 \%$ & \\
\hline \multirow{2}{*}{ Parity $(n=146)$} & $\mathbf{P}=\mathbf{0}$ & $29 \%$ & $28 \%$ & $38 \%$ & \multirow{2}{*}{0.30} \\
\hline & $\mathbf{P}>\mathbf{0}$ & $71 \%$ & $72 \%$ & $63 \%$ & \\
\hline \multirow{2}{*}{$\begin{array}{c}\text { Language } \\
(\mathrm{n}=119 *)\end{array}$} & English & $47 \%$ & $61 \%$ & $48 \%$ & \multirow{2}{*}{0.21} \\
\hline & Non English & $34 \%$ & $39 \%$ & $53 \%$ & \\
\hline \multirow{2}{*}{$\begin{array}{c}\text { Education } \\
(\mathrm{n}=104 *)\end{array}$} & High School Grad & $48 \%$ & $35 \%$ & $34 \%$ & \multirow[b]{2}{*}{0.86} \\
\hline & $\begin{array}{l}\text { Non High School } \\
\text { Grad }\end{array}$ & $23 \%$ & $65 \%$ & $66 \%$ & \\
\hline \multirow{2}{*}{$\begin{array}{l}\text { Marriage } \\
\left(\mathrm{n}=116^{*}\right)\end{array}$} & Married & $24 \%$ & $26 \%$ & $40 \%$ & \multirow{2}{*}{0.16} \\
\hline & Not Married & $55 \%$ & $74 \%$ & $60 \%$ & \\
\hline
\end{tabular}

*Incomplete data resulting in $\mathrm{n}<146$

\section{Error reduction}

A total of 547 visits were conducted for 153 high risk infants with a median of 3 visits and a range of 2-8 visits per infant. Of the 153 infants, 48 infants had no errors. Of the 105 infants who had errors, 82 were error free by their final visit and 23 had at least one error on their last visit.

A total of 282 errors were found in the 105 high risk infants, of the 282 errors found, $61 \%$ were appointment errors (median per infant 1, range 0-7), 5\% were equipment errors (median per infant 0 , range $0-2$ ), $18 \%$ were medication errors (median per infant 0 , range $0-5$ ) and $16 \%$ were feeding errors (median per infant 0 , range 0 -3). The figure reflects the total number of errors encountered across visits (see table 4). 
Table 4: Number of Errors

\begin{tabular}{|c|c|c|c|c|c|c|c|c|c|}
\hline Types of Errors & $\begin{array}{c}\text { Visit } \\
\# \mathbf{1} \\
(\mathrm{n}=153)\end{array}$ & $\begin{array}{c}\text { Visit } \\
\# \mathbf{2} \\
(\mathrm{n}=153)\end{array}$ & $\begin{array}{c}\text { Visit } \\
\# 3 \\
(\mathrm{n}=113)\end{array}$ & $\begin{array}{c}\text { Visit } \\
\# 4 \\
(n=64)\end{array}$ & $\begin{array}{c}\text { Visit } \\
\# 5 \\
(\mathrm{n}=34)\end{array}$ & $\begin{array}{c}\text { Visit } \\
\# \mathbf{6} \\
(\mathrm{n}=22)\end{array}$ & $\begin{array}{c}\text { Visit } \\
\# 7 \\
(\mathrm{n}=6)\end{array}$ & $\begin{array}{c}\text { Visit } \\
\begin{array}{c}\text { (8) } \\
(\mathrm{n}=2)\end{array}\end{array}$ & $\begin{array}{c}\text { Total } \\
(\mathrm{n}=153)\end{array}$ \\
\hline Appointment & 88 & 40 & 29 & 14 & 0 & 0 & 0 & 0 & 171 \\
\hline Equipment & 12 & 2 & 0 & 0 & 0 & 0 & 0 & 0 & 14 \\
\hline Medicine & 23 & 13 & 13 & 1 & 2 & 0 & 0 & 0 & 52 \\
\hline Feeding & 28 & 9 & 4 & 1 & 3 & 0 & 0 & 0 & 45 \\
\hline Total & 151 & 64 & 46 & 16 & 5 & 0 & 0 & 0 & 282 \\
\hline
\end{tabular}

When comparing the first and last visit error for each infant, there was a significant reduction in both homecare and healthcare utilization errors $(\mathrm{p}<0.001)$, there was also a temporal trend to error reduction when looking at the domains for appointment $(\mathrm{p}<0.001)$, feeding $(\mathrm{p}<0.001)$, medication $(\mathrm{p}<0.001)$ and equipment $(\mathrm{p}<0.05)$ individually (see figure 5$)$.

Figure 3: Graphical Representation of Error Reduction Over Visits

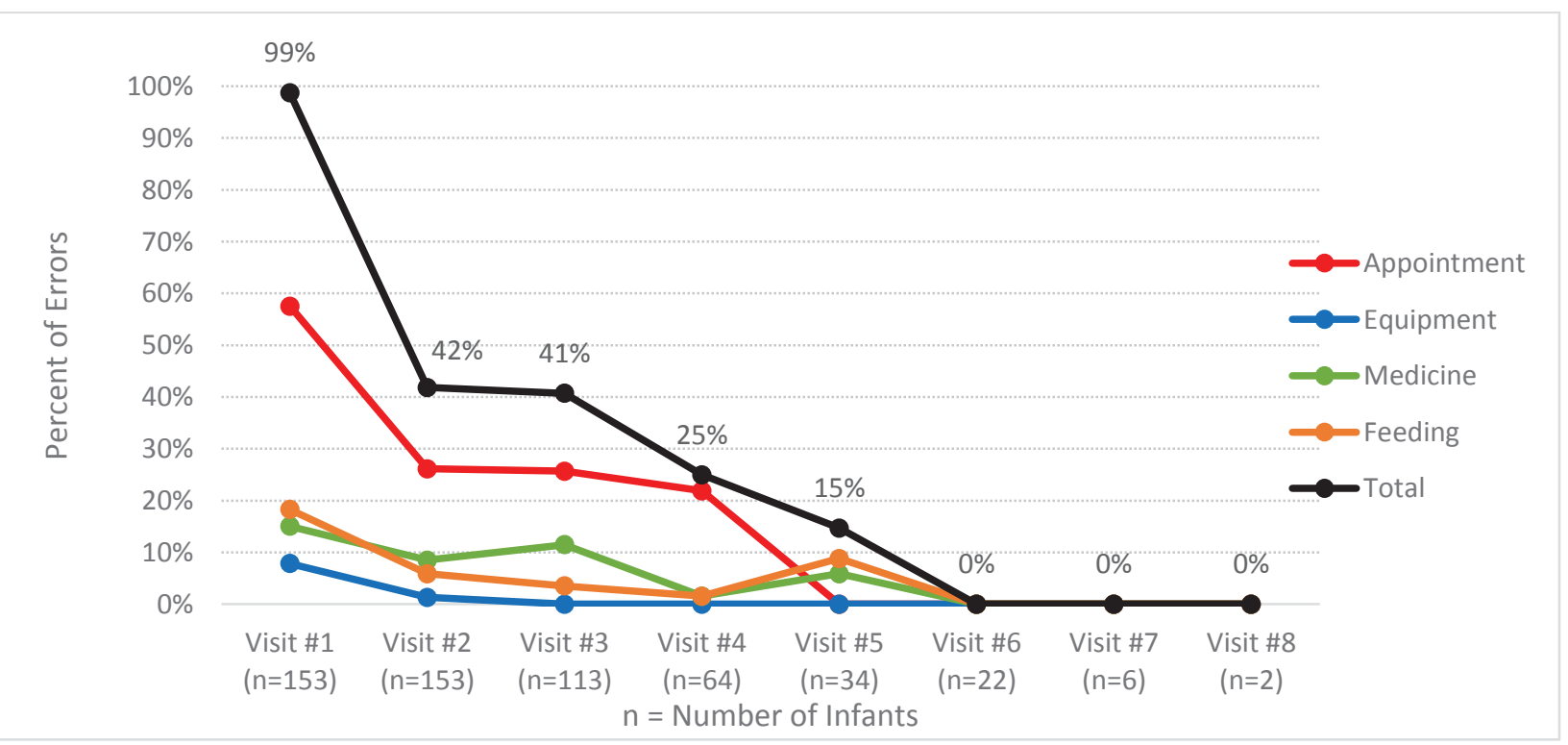




\section{Discussion}

Supporting high risk infants and their caregivers during the transition from NICU to home provides a powerful opportunity to prevent morbidity and mortality. PNP home visits reduce both home care and healthcare utilization errors in high risk infants, particularly with regard to ensuring proper follow up appointments, appropriate feeding regimens, medication administration, and home durable equipment use.

Maternal race, ethnicity, level of education and language have been reported as barriers to optimal healthcare utilization and care errors (Ames, 2007; Catlett et al., 1993; MiquelVerges, Donohue, \& Boss, 2011 Roberts et al., 2008; Samueis-Kalow, Stack, \& Porter, 2013; Sparks, 2009; Yu et al., 2006). However, this study did not find an association between caregiver and infant socio-demographic factors and errors. All high risk infants, independent of sociodemographic factors, gestational age, and length of NICU stay were equally likely to have home care and healthcare utilization errors. Our study results suggest that all high risk infants and their caregivers are susceptible to home care and healthcare utilization errors regardless of their sociodemographic status.

Errors that were identified and assessed in the first visit significantly decreased when compared to the last visit. The significant error reduction found for infants was likely due to PNPs' targeted interventions towards caregiver individual needs after assessing deviations from care plans.

This study demonstrates that there is an opportunity to improve high risk infant healthcare utilization errors and as they transition from NICU to home. The most frequently encountered errors were appointment errors. PNPs ameliorated appointment errors by identifying and responding to missed appointments, by re-scheduling appointments, making needed 
referrals, and communicating the importance of the appointments to caregivers. If these appointment errors had not been discovered, infants would have missed valuable follow up opportunities. Identification and intervention of appointment errors can prevent unnecessary use of emergency and urgent care services, and ensures infants get the specialized care they need to prevent morbidities such as blindness, cerebral palsy, respiratory syncytial virus, neurodevelopmental delay and failure to thrive (American Academy of Pediatrics, 1998).

The reduction in feeding errors was due to PNPs assessing feeding in the home and working with caregivers to optimize infant nutrition by customizing feeding plans, giving printed mixing and feeding instructions, and prescribing formula as needed. Close monitoring and individualization of feeding follow up has the potential to improve growth and may help infants avoid office visits and re-hospitalizations for weight loss and failure to thrive.

PNPs also identified and corrected medication errors in the home. The number of medication errors encountered in the final home visits was reduced from the number found in the initial visits. This reduction is thought to be due to PNP demonstrating correct medication preparation and administration for caregivers and prescribing needed medications that caregivers had not obtained. Each encountered medication error led to a significant reduction in potential morbidity.

Each equipment error was corrected by the final home visit. PNPs addressed equipment errors by demonstrating proper use of home medical equipment and aiding in obtaining the necessary home equipment. Intervention with regard to equipment errors may have prevented rehospitalization and unnecessary use of urgent and emergent care services.

High risk infant health outcomes stand to improve from in home PNP support that addresses and may even prevent home care errors. Such support also fosters comprehensive 
follow-up critical to the health outcomes of high risk infants. The scope of practice of PNPs makes them ideal providers of this needed comprehensive, cost effective support after NICU discharge.

To our knowledge this is the first prospective study to identify high risk infants' home care and healthcare utilization errors and show a significant reduction in these errors during the transition from the NICU to home.

\section{Limitations}

While it seems feasible that PNP led interventions were the primary factor responsible for error reduction, there may have been other factors that contributed. This prospective observational study did not control for other support that infants received, including public health nurse visits post NICU discharge. However, PNPs were the first home provider to see the infant and caregiver post NICU discharge. PHN home visit nurse notes were not accessible to the pediatrician or the home follow-up PNPs secondary to PHN notes not being a part of the patients medical record, thus there was no way of PNPs knowing if the PHNs found an error in any domain unless the PHN contacted the PNP via hospital e-mail or direct phone. PHN's did contact PNPs if they needed guidance with regard to the resolution of an error that was within their scope of practice or if they needed the PNP to intervene due to the PNPs broader scope of practice. Errors that were identified by the PHN and made aware to the PNP following the same infant and caregiver were not counted as an error for this study.

\section{Implications for Nursing Practice}

Understanding home care and health care utilization errors provides an opportunity to improve health care across transitions for high risk populations. Understanding why caregivers miss appointments, prepare and administer feedings and medications incorrectly and lack home 
medical equipment allows providers to support caregivers in supporting infants. This study could have significant impact on home visitation for high risk infants by introducing the role of a PNP in conjunction with a public health nurse for home visitation. Public health nursing programs across the nation may be able to utilize the identification process of errors in high risk infants and other vulnerable populations to help reduce the errors that occur with facilitation of a primary care provider if a PNP is unavailable.

PNP home visitation of high risk infants can play a key role in ensuring that the Institute for Healthcare Improvements triple aim which consists of improving the experience of care, improving the health of populations, and reducing per capita costs of health care is implemented (Berwick, Nola, \& Whittington, 2008). PNPs are ideally suited to identify and reduce home care errors and improve high risk infant health outcomes. PNP led home follow-up improves prevention, and coordination of services and has played a vital role in infant health promotion and disease prevention (Martinez, Schreiber, \& Hartman, 1991; Vasquez, Pitts, \& Mejia, 2008).

\section{Conclusion}

In summary, study findings have advanced our understanding of the types of errors that are encountered by high risk infants and their caregivers who are making the difficult transition from the NICU to home. It is important to understand these errors so that the resources and expertise needed to address them can be anticipated. Study findings have also increased our knowledge of PNPs interventions in response to the home care and healthcare utilization errors. Knowledge of errors and interventions used to reduce them is essential to being able to bridge errors and interventions with patient outcomes. 


\section{References}

Ames, N. (2007). Improving underserved children's access to health care: Practitioners' views. Journal of Child Health Care: For Professionals Working with Children in the Hospital and Community, 11(3), 175-185.

American Academy of Pediatrics (2008). Committee on Fetus and Newborn. Hospital discharge of the high-risk neonate. Pediatrics, 122:1119-1126.

American Academy of Pediatrics (1998). Committee on Fetus and Newborn. Hospital discharge of the high-risk neonate. Pediatrics. 1998;102(2 pt 1):411-417.

Banfield, B. (2011). Environment: A perspective of the self-care deficit nursing theory. Nursing Science Quarterly, 24(2), 96-100.

Berbiglia, V. (2011). The self-care deficit nursing theory as a curriculum conceptual framework in baccalaureate education. Nursing Science Quarterly, 24(2), 137-145.

Berwick, D. M., Nolan, T. W., \& Whittington, J. (2008). The triple aim: care, health, and cost. Health Affairs, 27(3), 759-769.

Brooten, D., Naylor, M., York, R., Brown, L., Roncoli, M., Hollingsworth, A., . . . . Jacobsen, B. (1995). Effects of nurse specialists transitional care on patient outcomes and cost: results of five randomized trials. American Journal of Managed Care, 1:35-41.

Cady, R. G., Kelly, A. M., Finkelstein, S. M., Looman, W. S., \& Garwick, A. W. (2014). Attributes of advanced practice registered nurse care coordination for children with medical complexity. Journal of Pediatric Health Care, 28(4), 305-312.

Catlett, A., Thompson, Jr, R., Johndrow, D., \& Boshkoff, M. (1993). Risk status for dropping out of developmental follow up for very low birth weight infants. Public Health Reports (1974-), 108(5), 589-594. 
Cohen, S. M., Arnold, L., Brown, L., \& Brooten, D. (1991). Taxonomic classification of transitional follow-up care nursing interventions with low birthweight infants. Clinical Nurse Specialist, 5(1), 31-36.

Duderstadt, K. (2008). Medical home: Nurse practitioners' role in health care delivery to vulnerable populations. Journal of Pediatric Health Care, 22(6), 390-393.

Evans, S. , Preston, F. , Daly, A. , Ashmore, C. , Holden, C. , et al. (2012). Home enteral tube feeding in children with inherited metabolic disorders: A review of long-term carer knowledge and technique. Journal of Human Nutrition and Dietetics : The Official Journal of the British Dietetic Association, 25(6), 520-525.

Glanz, K. , Burke, L., Rimer, B., (2011). Health behavior theories. In J. B. Butts \& K. L. Rich, K.L. (Eds.). Philosophies and theories for advanced practice nursing (pp. 143163). Sudbury, MA: Jones \& Bartlett Learning.

Guralnick, M. (2011). Why early intervention works a systems perspective. Infants \& Young Children, 24(1), 6-28.

Hartweg, D. L. (1991). Dorothea Orem: Self-care deficit theory. Location: (Newbury Park, CA) Sage Publications.

High Risk Infant Follow-up Quality of Care Initiative. (2012). California Children's Services and California Perinatal Quality Care Collaborative High Risk Infant Follow-up Quality of Care Initiative Manual of Definitions. Retrieved from website: https://www.ccshrif.org on October 4, 2014.

Hulsey, T., Hudson, P., Pittard, W. (1994). Predictors of hospital post-discharge infant mortality: implications for high-risk infant follow-up efforts. Journal of Perinatology. May-Jun;14(3):219-25. 
Institute of Medicine (US). (2011). Committee on the Robert Wood Johnson Foundation Initiative on the Future of Nursing. The future of nursing: Leading change, advancing health. National Academies Press.

Institute of Medicine. (2003). In: K. Adams \& J. M. Corrigan (Eds.), Priority Areas for National Action: Transforming Health Care Quality. National Academies Press.

John, J. (2005). Preventing medication errors at home. Journal of Pharmacy Practice, 18(3), 141144.

Looman, W. S., Presler, E., Erickson, M. M., Garwick, A. W., Cady, R. G., Kelly, A. M., \& Finkelstein, S. M. (2013). Care coordination for children with complex special health care needs: The value of the advanced practice nurse's enhanced scope of knowledge and practice. Journal of Pediatric Health Care, 27(4), 293-303.

Martin, J., \& Osterman, M. (2013) Centers for Disease Control and Prevention, National Center for Health Statistics. Preterm Births: United States, 2006 and 2010. Retrieved from: http://www.cdc.gov/mmwr/preview/mmwrhtml/su6203a22.htm?s_cid=su6203a22_w

Martinez, N. H., Schreiber, M. L., \& Hartman, E. W. (1991). Pediatric nurse practitioners: Primary care providers and case managers for chronically ill children at home. Journal of Pediatric Health Care, 5(6), 291-298.

Miquel-Verges, F., Donohue, P., \& Boss, R. (2011). Discharge of infants from nicu to latino families with limited english proficiency. Journal of Immigrant and Minority Health, 13(2), 309-314.

Murphy, M. A. (1990). A brief history of pediatric nurse practitioners and NAPNAP 1964-1990. Journal of Pediatric Health Care, 4(6), 332-337.

Naylor, M. D., Bowles, K. H. \& Brooten, D. (2000), Patient Problems and Advanced Practice 
Nurse Interventions During Transitional Care. Public Health Nursing, 17, 94-102.

Naylor, M. D., Brooten, D., Campbell, R., Jacobsen, B. S., Mezey, M. D., Pauly, M. V., \& Schwartz, J. S. (1999). Comprehensive discharge planning and home follow-up of hospitalized elders: a randomized clinical trial. Journal of the American Medical Association, 281(7), 613-620.

Neal-Boylan, L., Mager, D. R., \& Kazer, M. W. (2012). Nurse Practitioners in Home Health Care An Update. Home Health Care Management \& Practice, 24(4), 193-197.

Roberts, G., Howard, K., Spittle, A., Brown, N., Andeson, P., et al. (2008). Rates of early intervention servies in very preterm children with developmental disabilities at age 2 years. Journal of Paediatrics and Child Health, 44(5), 276-280.

Running, A., \& Walker, T. (1999). Using nurse practitioners in home care: A report of two pilot projects. Home healthcare nurse, 17(11), 731-738.

Samuels-Kalow, M. E., Stack, A. M., \& Porter, S. C. (2013). Parental language and dosing errors after discharge from the pediatric emergency department. Pediatric emergency care, 29(9), 982-987.

Sparks, P. (2009). One size does not fit all: an examination of low birthweight disparities among a divers set of racial/ethnic groups. Maternal Child Health Journal, 68(13), 769-779.

Taylor, S. , \& Cox, K. (2005). Orem's self-care deficit nursing theory: Pediatric asthma as exemplar. Nursing Science Quarterly, 18(3), 249-257.

Vasquez, E. P., Pitts, K., \& Mejia, N. E. (2008). A model program: neonatal nurse practitioners providing community health care for high-risk infants. Neonatal Network, 27(3), 163169. 
Wang, C., Guttmann, A. , To, T. , \& Dick, P. (2009). Neighborhood income and health outcomes in infants: How do those with complex chronic conditions fare?. Archives of Pediatrics \& Adolescent Medicine, 163(7), 608-615.

World Health Organization. (2009). WHO/UNICEF Joint Statement: home visits for the newborn child: a strategy to improve survival. Geneva: World Health Organization, USAID and Save the Chilren. Retrieved from: http://whqlibdoc.who.int/hq/2009/WHO_FCH_CAH_09.02_eng.pdf?ua=1

Yu, S., Huang, Z., Schwalberg, R., \& Nyman, R. (2006). Parental english proficiency and children's health services access. American Journal of Public Health, 96(8), 1449-1455. 


\section{APPENDIX A}

CCS HRIF PROGRAM MEDICAL ELIGIBILITY CRITERIA

UPDATED 01/2014

Data should be collected on infants/children under three years of age who meet California Children's Services (CCS) HRIF medical eligibility criteria and who met CCS medical eligibility criteria for Neonatal Intensive Care Unit (NICU) care OR had a CCS eligible medical condition at some time during their stay in a CCS-approved NICU, even if they were never a CCS client. Infants are medically eligible for the HRIF Program when the infant:

\begin{abstract}
Met CCS medical eligible criteria for NICU care, in a CCS-approved NICU, regardless of length of stay, (as per Number Letter 05-0502, Medical Eligibility in a CCS-approved NICU). NOTE: Medical eligibility includes neonates who require direct admit to a CCS-approved PICU, who are never admitted to a CCS-approved NICU, but who otherwise meet all medical eligibility criteria for HRIF services.
\end{abstract}

Had a CCS eligible medical condition in a CCS-approved NICU,

OR regardless of length of stay, (as per California Code of Regulations, Title 22, Section 41800 through 41872, CCS Medical Eligibility Regulations).

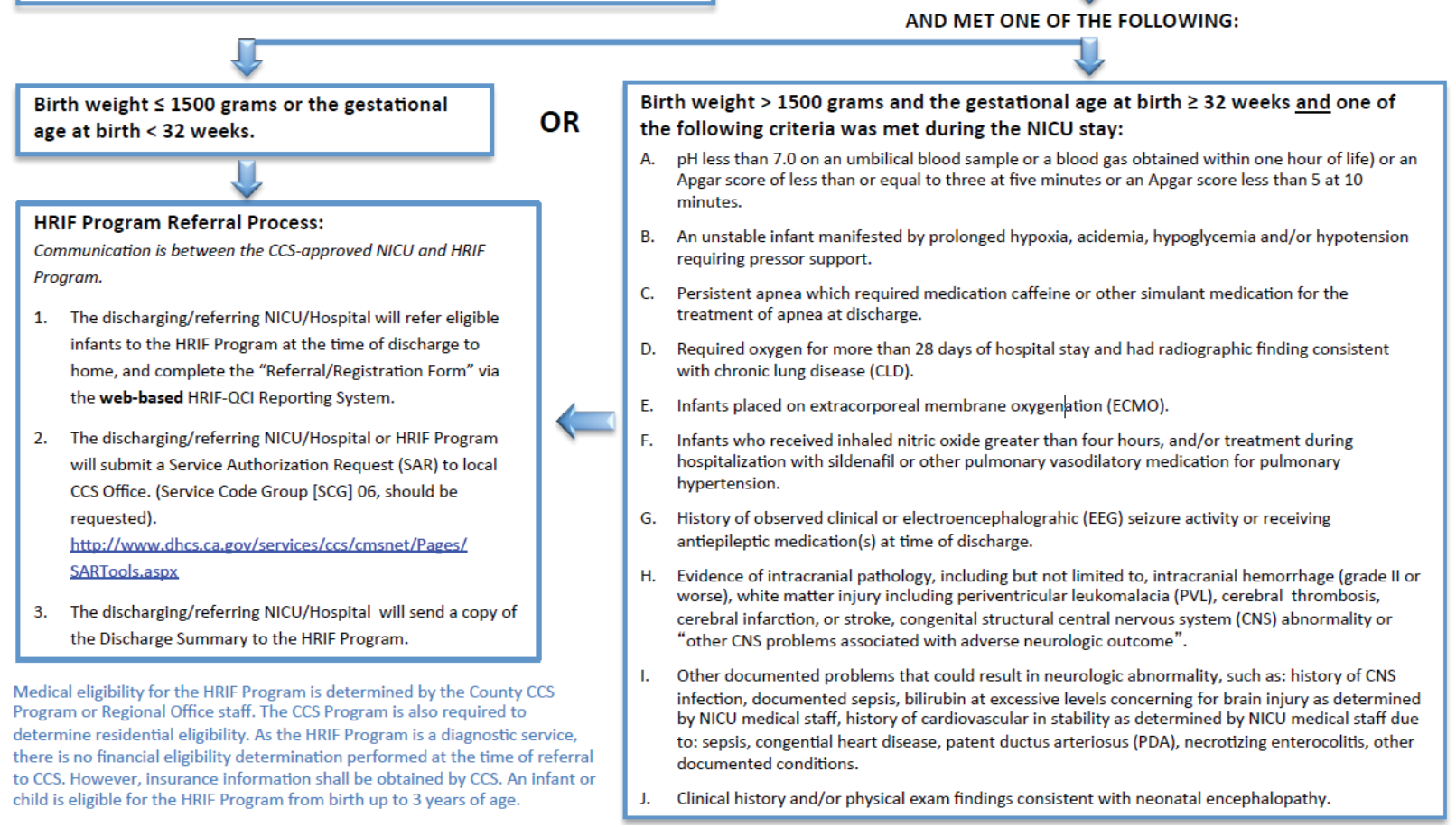




\section{APPENDIX B \\ SCVHHS BRIDGE DATA COLLECTION SHEET}

1. Number (unique identifier):

2. Diagnoses:

3. Visit \#:

4. Quality Measures:

a. Medications:

Verbalization of correct: Name of medication, Dose, Timing, and Method of administration

Demonstration of competency

Caregiver comments

b. Discharge feeding management: type of milk or formula, caloric density, mixing instructions, breastfeeding, expressed breast milk feeding, fortification of expressed breast milk, timing of feedings

c. Equipment-Type (eg oxygen saturation monitor, feeding pump and G-tube)

Is the equipment present in home (Yes / No)

Verbalization of competency

Demonstration of competency

d. Pediatrician and subspecialist appointments ( provider, time, date, location (Yes / No) Verbalization of competency

e. Appropriate Health Care Utilization

Verbalization of health care system access knowledge

Action taken (eg appointments scheduled, ER visit, provided information) 